\title{
3D Screen Printing Enables Application of Integrated QR Codes on Pharmaceutical Dosage Forms in Mass Production - A Game Changer
}

\author{
Marcel Enke', Klaus Kühne ${ }^{1,2}$, Stefan Seyferth ${ }^{3}$, Dagmar Fischer ${ }^{3}$ and Achim Schneeberger ${ }^{1,2 *}$ \\ ${ }^{1}$ Laxxon Medical GmbH, Germany \\ ${ }^{2}$ Laxxon Medical Corp, USA
}

${ }^{3}$ Division of Pharmaceutical Technology, Department of Chemistry and Pharmacy, Friedrich-Alexander University Erlangen, Germany

*Corresponding author: Achim Schneeberger, Laxxon Medical GmbH, Hans-Knöll-Str. 6, 07745 Jena, Germany and Laxxon Medical

Corp, 135 E $57^{\text {th }}$ Street, $14^{\text {th }}$ floor, NewYork, NY 10022, USA

\section{ARTICLE INFO}

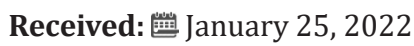

Published: 幽 February 03, 2022

Citation: Marcel Enke, Klaus Kühne, Stefan Seyferth, Dagmar Fischer, Achim Schneeberger. 3D Screen Printing Enables Application of Integrated QR Codes on Pharmaceutical Dosage Forms in Mass Production - A Game Changer. Biomed J Sci \& Tech Res 41(4)-2022. BJSTR. MS.ID.006630.

\begin{abstract}
Quick Response (QR) codes have shown enormous potential to improve the flow of information from industry to providers and patients, by enabling access to supplemental safety data, potential drug interactions, among numerous other use-cases in real-time. Advances in 3D printing technology, such as 3D screen printing (3DSP), have allowed for mass customization of pharmaceuticals down to the single-tablet level. The integration of in-drug labeling of pharmaceuticals with unique identifying information, such as QR codes, has been shown to reduce medication errors, improve patient safety, and provide a viable mechanism for remote patient monitoring and identifying counterfeit drugs. This review investigates the progress that has been made in the last few years in using QR codes for drug labeling, with a specific focus on the potential for using 3D printing technology, including screen printing, to expedite $\mathrm{QR}$ code integration at the single tablet level.
\end{abstract}

Keywords: QR Code; 3D Printing, Additive Manufacturing; 3D Screen Printing; Remote Patient Monitoring; Counterfeit Medicines; Medication Safety

\section{Introduction}

Quick Response (QR) codes have been in use since the 1990s. They were initially developed as a way to track components of vehicles at Denso Wave (a Japanese company and a subsidiary of Toyota) through the supply chain [1]. Since then, their use has steadily increased. The widespread availability and decreasing costs of entry for mobile devices such as smartphones and tablets that can quickly read QR codes have significantly contributed to their quick adoption. A QR code is simply a two-dimensional barcode. It looks like a crossword puzzle with black and white squares called modules. The square grid-like design allows for an increased storage of information compared to a standard one-dimensional bar code. QR codes can store about 7,000 digits (around 4,000 characters) of information such as linking to a specific website. It includes a high-level of integrated redundancy, allowing for the QR code to be readable even if a substantial portion (around 30\%) of the code has been damaged. Today, QR codes are ubiquitous and can be found everywhere from construction through social media to banking. The flexibility of their design and the ability to quickly update their information makes them easily amenable to a wide range of applications, including healthcare.

QR codes have already proven to be highly beneficial in healthcare such as patient tracking, drug safety and many other use- 
cases (Figure 1). They can be used to digitally store product safety, medication interactions, among others, as well the ability to update this information in real-time for patients and health care providers. Moreover, implementing QR codes directly on dosage forms is an efficient means for combating counterfeit medicines. This review investigates the potential for using QR codes in medicine, with a special focus on their application for pharmaceuticals using 3D printing technology, including screen printing.

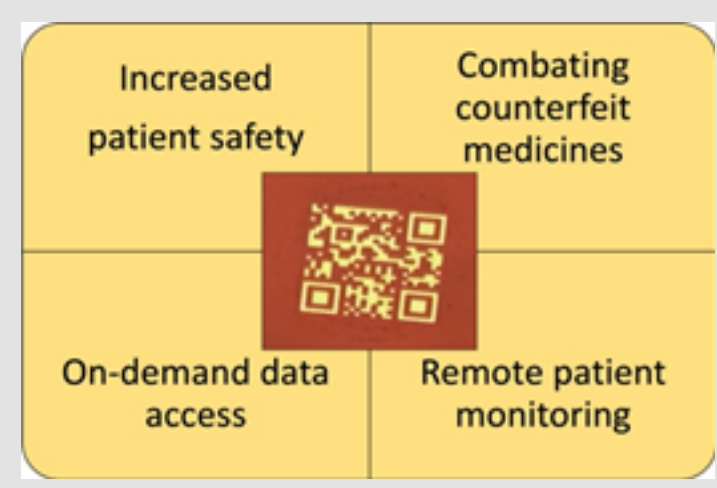

Figure 1: Advantages of using QR codes for pharmaceutical dosage forms in mass production.

\section{Tablet Level Labeling}

Additive manufacturing is an increasingly disruptive force in pharmaceutical development [2-4]. 3D printing (3DP) approaches enable custom geometry, size, content, and release characteristics of active pharmaceutical ingredients (API). At the same time, digital modelling has allowed for a new level of freedom in drug design. These opportunities have led to a boom in 3DP technologies for pharmaceutical development. Although conceptually similar, each uses its own method to deposit each layer of material and cure it [59]. There are two primary approaches for additive manufacturing: personalized drug development and production for the mass market. The ability to produce custom drugs for the mass market has a lot of appeal for responding to changing needs at scale and have been a major focus of recent technological developments.

However, concerns over scalability of 3DP technologies, low mechanical resistance, low printing resolution and limited material choices have so far limited their practical implementation $[10,11]$. Currently, only a single 3D printed pharmaceutical, the antiepileptic drug Spritam ${ }^{\circledR}$ is FDA approved and marketed [12]. The first types of 3DP pharmaceuticals were developed using continuous inkjet printing [13]. Since then, advances in inkjet printing [14,15] as well as newer technologies, most notably: extrusion-based printing (fused deposition modelling (FDM), and screen printing) [16-18], powder-based printing (powder bed and powder jetting) [19-21], selective laser sintering (SLS) printing [22], have aimed to print instead pharmaceuticals for the mass market. Among the 3DP technologies, extrusion-based printing, such as FDM, direct ink writing (DIW), and most recently 3D screen printing (3DSP), have shown the greatest promise due to their low cost and flexibility.

All extrusion-based 3D printers have two principal components

1. The extruder (which deposits the precise quantity of material over a specific distance) and

2. The positioning system.

The pintable material (i.e., ink) in the form a viscous melt or liquid is extruded through a nozzle (in case of FDM and DIW) or through a tissue (in case of 3DSP). While the nozzle gets only a single printer, the tissue, as a function of its area, mediates extrusion of paste for multiple objects at the same time. The newkid on the extrusion-based printing block is 3DSP. 3DSP is a type of extrusion printing that uses a screen mesh to transfer a semisolid, API containing paste onto a substrate, except in areas made impermeable to the paste by a blocking stencil. The deposited layer is then dried. The next layer is printed precisely on top of the previous one after lifting the screen by the dried layer thickness. 3DSP has the potential for mass customization enabling the buildup of thousands of units per screen simultaneously. The number of units printed simultaneously is largely defined by the ratio of screen size to unit size. This differentiates screen printing from other 3DP technologies, whose capacity is limited by the number of printing heads [10,23]. Exentis Group [24], based in Switzerland has developed the patented 3DSP technology for a broad range of industrial applications, such as renewable energy sources, automotive industry, aerospace, and biotechnology. The Exentis 3DSP enables the production of several hundred items with the option for a mass production of an upscaling to several million a day. Laxxon Medical Corp holds the exclusive worldwide license to explore this 3DSP technology for all kind of pharmaceutical applications.

Laxxon Medical Corp. is developing a screen-printing approach for printing pharmaceuticals for the mass market. This approach is based on a classic flatbed screen printing that is widely used in industrial applications [25,26]. Proof-of-concept work with 3DSP (SPID®-Technology) has shown that it is possible inter alia to print $\mathrm{QR}$ codes directly on tablets as small as $\mathrm{r}=5 \mathrm{~mm}$ during and as integrated part of the manufacturing process (Figure 2). Using this approach, the QR code is part of a one-stop manufacturing process of the tablet. 3DSP could also be used to print API containing QR codes and/or inks which make the tablet easier to identify, harder to counterfeit and increases product safety. There are at least two ways that a QR code can be printed directly on tablets. The first is to print the code on top of the tablet using different kinds of inks. Inkjet printing for example has previously been shown as a viable strategy for printing QR codes directly on oral dosage formulations. 
Work by Edinger et al. showed that it was possible to print specific amounts of the neuroleptic drug haloperidol as a QR code on an edible orodispersible substrate [27]. Here, researchers used the QR code to identify the API, dose, patient name, administration route, expiration date, manufacturer ID and batch number as a proof-ofconcept. Further work by Trenfield et al. combined both 2D and
3D inkjet printing technologies in a single-step manufacturing process to create drug-loaded tablets with a combined QR code identifier that used anti-counterfeit ink [28]. In both cases, a personalized medicine approach was investigated instead of a mass customization strategy, but there is clear potential for both strategies.
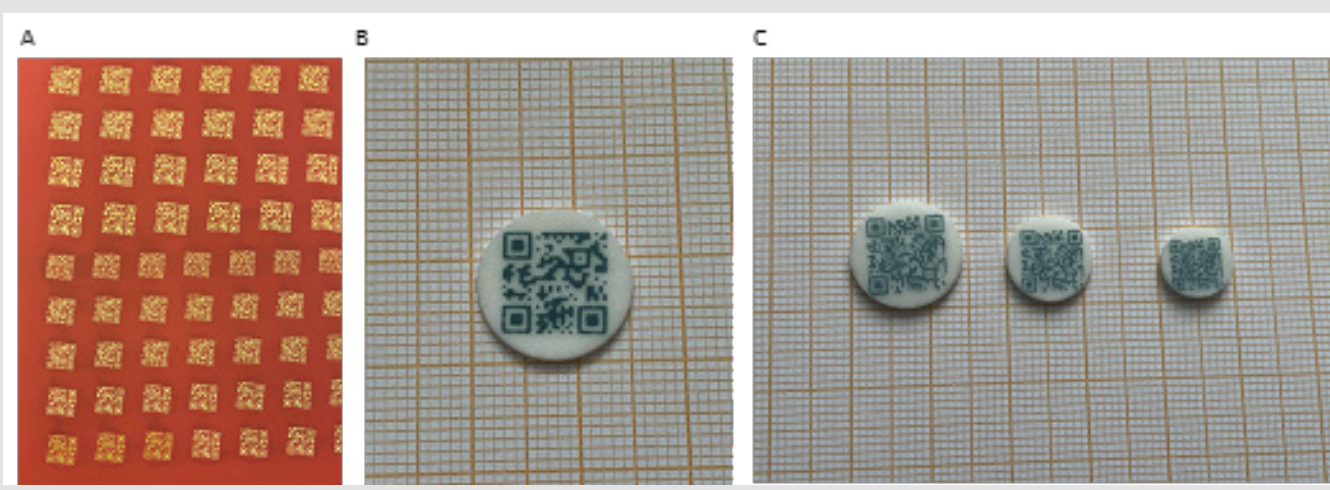

Figure 2: 3D Screen Printed Tablets with integrated QR Code.

A. QR code screen with different sizes (diameter) and QR code designs used within the 3D Screen Printing Unit EX 301i.

B. Tablets were printed with 92 levels of each $0.025 \mathrm{~mm}$. Afterwards, at the end of the manufacturing process, 2 additional layers with the QR code pattern were applied. A mixture of black and blue (2:1) commercial food dye was utilized for coloring the QR-code. Example QR code printed on a $9.5 \mathrm{~mm}$ size tablet.

C. More complex QR Code (allows larger amount of data) were printed on different sized tablets 14.5, 11.5, 9.5 mm (from left to right).

The ability to print a QR code directly on the tablet would be a major benefit for patients and health care professionals (HCPs). It would allow them to confidently identify the medication and access all relevant product information, even without the need of any secondary packing information. This could be helpful to older patients who may be taking multiple medications, and who may separate their pills into pill boxes without any identifying information. It is also of great interest to patients who may be visually impaired and have trouble identifying their medication even with the packaging information available [29]. Also, patient safety could increase through automated dispensing enabled through this QR code marking [30]. This is especially important in the hospital setting, a situation characterized by frequent medication changes and of increasing patient turnover. Checking drug data against patient data, both in an electronic format and at the point of dispense, could maximize patient safety.

An alternative to printing the $\mathrm{QR}$ code on the tablet itself is to embed the code into the tablet [31]. One such proposal used grooving to offset the surface inward to create light and dark regions resulting from ambient occlusion. This could allow for tablets with rough surface structures as well as a wide range of surface colors to have a readable QR code. While conceivable, the outlined solution is complex as its implementation requires the sequential application of two technically different processes. In contrast, 3DSP is capable of yielding tablets with embedded QR codes by applying two additional steps of the very same production process. First, a negative print is administered on top of the printed tablet's body using the "negative" screen, then resulting recesses are filled using the "positive" screen and a colored paste. Requirements are well within the scope of the screen-printing technology, namely two more screens and two pastes with different colors. Where needed, the $\mathrm{QR}$ code could be sealed by applying a thin layer of transparent paste.

\section{Improving Medication Safety}

Product safety communication in medicines is an integral, interactive exchange among regulatory authority, HCPs, and patients, so that all parties understand the product risks. The backbone of this communication starts at the level of defining what a medicine is. The safety of pharmaceuticals, especially regarding the testing, approval and manufacture of drugs, pharmacovigilance, the distribution of drugs, and the monitoring of drug traffic for NCAs is defined in national laws, e.g., Section 1 of the German Medicines Act (AMG) [32]. This regulation covers what qualifies a product as a pharmaceutical, a generic, as well as counterfeit medicines. 
Patients and HCPs are most often informed of product risks in statutory information such as that contained in the product information (i.e., the summary of product characteristics (SmPC), package leaflet (PL) and the labelling of the packaging) and public assessment reports [33]. A key limitation of this content is the speed for the implementation of updates. In this format, emerging safety issues discovered from NCAs and HCPs cannot be updated in real-time, leaving potentially vulnerable patients at a greater risk as a result of the inflexibility of this communication method for months, if not longer. Moreover, PLs and additional supplementary information provided with prescription medication can be easily lost or discarded by the patient.

Medication errors, incorrect dosages, and unintended drug interactions have reduced the effectivity of available care. Medication errors account for an estimated one-quarter of all healthcare errors [34], costing an estimated US\$42 billion annually worldwide according to the World Health Organization (WHO) [35]. Errors may happen while prescribing, transcribing, dispensing and administrating medication. A combination of look-alike/sound-alike medications, overwhelmed doctors and nurses, and the increasing complexity of patient care all contribute to an increased rate of mistakes. Patient error resulting from misunderstanding medication labels contributes significantly to avoidable hospitalizations. Up to $25 \%$ of all medication errors can be attributed to name confusion and 33\% to packaging and labeling confusion [36]. This issue is further complicated in older patients who may be taking prescription drugs and supplements simultaneously [37]. For example, warfarin, a common prescription blood anticoagulant, and Ginkgo biloba, a common herb supplement, can both reduce blood clotting. Taking these products together can increase chances of internal bleeding and stroke in some patients [38]. However, this level of information is very often missing from PL.

The European Union's Innovative Medicines Initiative WEBRADR has spent the last three years investigating how the influx of mobile devices, such as smartphones, could offer an ideal platform to improve communication of emerging safety issues [39]. Based on the progress in this arena, the European Medicines Agency (EMA) published guidelines on the use of QR codes for labeling and packaging of authorized medications in 2018 [40]. An early study from 2015 found that adding QR codes to the labels reduced medication errors in older patients taking multiple medications [41]. Further studies have looked at how medication errors may be reduced across different age groups. Recent work from Arizona State University undergraduates and senior citizens over 70 for example, similarly, found statistically reductions in medication errors for both groups when a QR code from the label was scanned prior to taking the medication [42].
Automated dispensing of medications in pharmacies and in hospital settings is a common way to provide medications to patients on-demand and to fill prescriptions close to the pointof-care. Automated dispensing cabinets and similar technologies aim to increase efficiency and reduce medication errors. There are several systemic reviews [43-45] which have found that automated dispensing was an effective way to decrease medication errors and improve patient management. Barcodes, and in many cases QR codes, can be read by these technologies. This efficiency could be bolstered by the addition of a QR code on the tablet itself, as this would allow for control at the single tablet level.

\section{Reliable Remote Patient Monitoring}

Patient adherence to their medication remains a substantial barrier in getting the most out of their prescribed treatment. An estimated $50 \%$ of patients on long-term therapies do not take their medications as prescribed [46]. Tight medication monitoring is also critical for medications which may be prone to misuse, such as those which are highly addictive. One popular example is the widespread use of medical cannabis. The prescribed dose of medical cannabis is highly individual, and the medication is prone to misuse, requiring close supervision by HCPs [47]. Recent publications on data-enriched edible pharmaceuticals (DEEP) used QR codes containing lipophilic cannabinoids, i.e., cannabidiol (CBD) and delta-9-tetrahydrocannabinol (THC), that were printed using a desktop inkjet 3D printer $[48,49]$. They printed a customized dose that could be readable by standard smartphone applications which remained readable after 8 weeks of storage under standard conditions. This approach shows the ability to use the QR code to directly label the substance at the single dosage unit level and track the patient's adherence to taking that prescribed dose. A further approach could be the development of specific apps for medication management that can scan $\mathrm{QR}$ encoded dosages prior to taking the medication [41,50-52]. Such apps have already shown promising results in improving compliance of elderly patients with heart disease [53] as well as for mental health interventions [54].

\section{Combating Counterfeit Medicines}

Counterfeit medicines make up an estimated US $\$ 200$ billion of the pharmaceuticals market annually, making it the largest identified fraud market globally [55]. In the industrialized world, estimates suggest that less than $1 \%$ of medicines sold are counterfeit [56]. However, in developing countries, these can be as high as $30 \%$. The statistics are further complicated by the increased number of consumers buying medication online, increasing the risk of obtaining counterfeit products from suppliers globally. In response, the United Nations (UN) under the auspices of the UN Office on Drugs and Crime (UNODC) started in 2014 a global campaign to raise awareness among consumers on the dangers of counterfeit goods and their link to organized crime [57]. 
The addition of QR codes to pharmaceuticals and pharmaceutical packaging has been suggested as a way to help reduce the number of counterfeit medicines in circulation at least as early as 2015 [58]. QR codes would be more difficult to replicate compared to standard text and image-based labeling used on pharmaceuticals. QR codes can be further equipped in anti-counterfeiting features, such as holograms [59] and fluorescent inks [60] which could help prevent falsified QR codes from appearing on the market. Drug-loaded biodegradable labels in the shape of $\mathrm{QR}$ codes have already been manufactured by casting the drug solution into prepared molds [59]. Although QR codes could be applied to both packaging and the drug dosage form, packaging labels are only an effective method if the drugs themselves are not repackaged. To decrease this risk there is an increased attention to "in-drug labeling" (i.e., direct tablet labeling) as an untapped tool to protect consumers from counterfeit medications [61]. Such in-drug labeling approaches would need to be ideally developed not only for solid drug formulations but also injectables and liquid formulations which may contain expensive and/or dangerous APIs that are high-interest targets for forgery.

\section{Outlook}

QR codes have a lot of potential to improve the way patients and HCPs interact with prescribed medications and treatments. Technical hurdles do remain for patients who may still be uncomfortable or have physical limitations with using QR codes and smart mobile devices. Additional training needs to be taken into consideration for these patients. It also should be remembered that the implementation of a $Q R$ code on pharmaceuticals would not be a complete replacement for existing patient leaflets. The basic information about the product still needs to be provided in a printed form to allow for patients to be able to access the information even if their smartphone is out of batteries. QR codes instead should be viewed as a way to augment this printed information with further details on medication interactions, explaining medical terms, and safety alerts that might be relevant to their specific treatment. The increased availability of 3D printing options for pharmaceuticals makes the addition of the QR code to the labeling and to the packing itself already within technical reach.

Safety concerns using QR codes could however continue to arise if the information linked to the QR code is either no longer in use or had been moved from its original online location. This can be mitigated by using dynamic QR codes instead of static QR codes. Regulations on the updating of online medical information must be in place to ensure that the information that is available to patients is the most accurate and up-to-date available. If there is one thing that has been highlighted by the COVID-19 pandemic more than anything, it is that the pace the medical information, including recommended treatments, can change overnight. Although this is an extreme example, all registered medications continue to be researched and have their safety information tracked after the patient information booklet has been printed. For all medicines, having the ability to update patients and doctors with this new information on-demand in an easy electronic way, would be invaluable.

\section{Author Contributions}

Writing-original draft preparation, K.K and M.E.; writing-review and editing, S.S. and D.F.; supervision, A.S. All authors have read and agreed to the published version of the manuscript.

\section{Acknowledgment}

Financial support of the Thüringer Aufbaubank (TAB; WE180124) is gratefully acknowledged.

\section{Conflicts of Interest}

K.K, M.E, and A.S. are employees of Laxxon Medical GmbH. D.F. and S.S. declare to have no competing financial interest.

\section{References}

1. QR Codes 101: A Beginner's Guide.

2. Beg S, Almalki WH, Malik A, Farhan M, Aatif M, et al. (2020) 3D Printing for Drug Delivery and Biomedical Applications. Drug Discovery Today 25: $1668-1681$.

3. Trenfield SJ, Awad A, Madla CM, Hatton GB, Firth J, et al. (2019) Shaping the Future: Recent Advances of 3D Printing in Drug Delivery and Healthcare. Expert Opinion on Drug Delivery 16: 1081-1094.

4. Khairuzzaman A (2018) Regulatory Perspectives on 3D Printing in Pharmaceuticals. In 3D Printing of Pharmaceuticals, In: Basit AW, Gaisford S (Eds.)., AAPS Advances in the Pharmaceutical Sciences Series, Springer International Publishing: Cham 31: 215-236.

5. Trenfield SJ, Madla CM, Basit AW, Gaisford S (2018) Binder Jet Printing in Pharmaceutical Manufacturing. In 3D Printing of Pharmaceuticals, In: Basit AW, Gaisford S, (Eds.)., AAPS Advances in the Pharmaceutical Sciences Series, Springer International Publishing: Cham 31: 41-54.

6. Awad A, Gaisford S, Basit AW (2018) Fused Deposition Modelling: Advances in Engineering and Medicine. In 3D Printing of Pharmaceuticals In: Basit AW, Gaisford S (Eds.)., AAPS Advances in the Pharmaceutical Sciences Series, Springer International Publishing: Cham 31: 107-132.

7. Firth J, Basit AW, Gaisford S (2018) The Role of Semi-Solid Extrusion Printing in Clinical Practice. In 3D Printing of Pharmaceuticals In: Basit AW, Gaisford S (Eds.)., AAPS Advances in the Pharmaceutical Sciences Series, Springer International Publishing: Cham 31: 133-151.

8. Awad A, Fina F, Goyanes A, Gaisford S, Basit AW (2020) 3D Printing: Principles and Pharmaceutical Applications of Selective Laser Sintering. International Journal of Pharmaceutics 586: 119594.

9. Robles Martinez P, Basit AW, Gaisford S (2018) The History, Developments and Opportunities of Stereolithography. In 3D Printing of Pharmaceuticals, In: Basit AW, Gaisford S (Eds.)., AAPS Advances in the Pharmaceutical Sciences Series, Springer International Publishing: Cham 31: 55-79.

10. Alhnan MA, Okwuosa TC, Sadia M, Wan KW, Ahmed W, et al. (2016) Emergence of 3D Printed Dosage Forms: Opportunities and Challenges. Pharm Res 33: 1817-1832. 
11. El Aita I, Ponsar H, Quodbach J (2019) A Critical Review on 3D-Printed Dosage Forms. CPD 24: 4957-4978.

12. West TG, Bradbury TJ (2018) 3D Printing: A Case of ZipDose ${ }^{\circledR}$ Technology - World's First 3D Printing Platform to Obtain FDA Approval for a Pharmaceutical Product. In 3D and 4D Printing in Biomedical Applications, In: Maniruzzaman M (Edt.)., Wiley-VCH Verlag GmbH \& Co. KGaA: Weinheim, Germany, p. 53-79.

13. Wu BM, Borland SW, Giordano RA, Cima LG, Sachs EM, et al. (1996) Solid Free-Form Fabrication of Drug Delivery Devices. Journal of Controlled Release 40: 77-87.

14. Kyobula M, Adedeji A, Alexander MR, Saleh E, Wildman R, et al. (2017) 3D Inkjet Printing of Tablets Exploiting Bespoke Complex Geometries for Controlled and Tuneable Drug Release. Journal of Controlled Release 261: 207-215.

15. Cader HK, Rance GA, Alexander MR, Gonçalves AD, Roberts CJ, et al. (2019) Water-Based 3D Inkjet Printing of an Oral Pharmaceutical Dosage Form. International Journal of Pharmaceutics 564: 359-368.

16. Khaled SA, Burley JC, Alexander MR, Roberts CJ (2014) Desktop 3D Printing of Controlled Release Pharmaceutical Bilayer Tablets. International Journal of Pharmaceutics 461: 105-111.

17. Goyanes A, Wang J, Buanz A, Martínez-Pacheco R, Telford R, Gaisford S, et al. (2015) 3D Printing of Medicines: Engineering Novel Oral Devices with Unique Design and Drug Release Characteristics. Mol Pharmaceutics 12: 4077-4084.

18. Pietrzak K, Isreb A, Alhnan MA (2015) A Flexible-Dose Dispenser for Immediate and Extended Release 3D Printed Tablets. European Journal of Pharmaceutics and Biopharmaceutics 96: 380-387.

19. Infanger S, Haemmerli A, Iliev S, Baier A, Stoyanov E, et al. (2019) Powder Bed 3D-Printing of Highly Loaded Drug Delivery Devices with Hydroxypropyl Cellulose as Solid Binder. International Journal of Pharmaceutics 555: 198-206.

20. Wang CC, Tejwani (Motwani) MR, Roach WJ, Kay JL, Yoo J, et al. (2006) Development of Near Zero-Order Release Dosage Forms Using ThreeDimensional Printing (3-DPTM) Technology. Drug Development and Industrial Pharmacy 32: 367-376.

21. Yu DG, Yang XL, Huang WD, Liu J, Wang YG, et al. (2007) Tablets With Material Gradients Fabricated by Three-Dimensional Printing. Journal of Pharmaceutical Sciences 96: 2446-2456.

22. Awad A, Fina F, Trenfield S, Patel P, Goyanes A, et al. (2019) 3D Printed Pellets (Miniprintlets): A Novel, Multi-Drug, Controlled Release Platform Technology. Pharmaceutics 11: 148.

23. Hsiao WK, Lorber B, Reitsamer H, Khinast J (2018) 3D Printing of Oral Drugs: A New Reality or Hype? Expert Opinion on Drug Delivery 15: 1-4.

24. Extensis Group.

25. (2001) Handbook of Print Media. In: Kipphan H (Edt.)., Springer Berlin Heidelberg: Berlin, Heidelberg.

26. Jurisch M, Studnitzky T, Andersen O, Kieback B (2015) 3D Screen Printing for the Fabrication of Small Intricate Ti-6Al-4V Parts. Powder Metallurgy 58: 339-343.

27. Edinger M, Bar-Shalom D, Sandler N, Rantanen J, Genina N (2018) QR Encoded Smart Oral Dosage Forms by Inkjet Printing. International Journal of Pharmaceutics 536: 138-145.

28. Trenfield SJ, Xian Tan H, Awad A, Buanz A, Gaisford S, et al. (2019) Trackand-Trace: Novel Anti-Counterfeit Measures for 3D Printed Personalized Drug Products Using Smart Material Inks. International Journal of Pharmaceutics 567: 118443
29. Almuzaini MA, Abdullah-Al-Wadud M (2018) A Review on Medication Identification Techniques for Visually Impaired Patients. In Proceedings of the $201821^{\text {st }}$ Saudi Computer Society National Computer Conference (NCC), IEEE: Riyadh, p. 1-6.

30. Fung EY, Leung B, Hamilton D, Hope J (2009) Do Automated Dispensing Machines Improve Patient Safety? CJHP 62(6): 516-519.

31. Kikuchi R, Yoshikawa S, Jayaraman PK, Zheng J, Maekawa T (2018) Embedding QR Codes onto B-Spline Surfaces for 3D Printing. ComputerAided Design 102: 215-223.

32.Amin-Farid A (2015) Definitionen Zu Pharmakovigilanz Und Arzneimitteltherapiesicherheit (AMTS). Arzneiverordnung in der Praxis 42.

33. Sharma R (2019) Monitoring Product Safety beyond Clinical TrialsIndustry Practices. Express Pharma.

34. Balkrishnan R, Patel I (2010) Medication Error Management around the Globe: An Overview. Indian J Pharm Sci 72: 539-545.

35. Donaldson LJ, Kelley ET, Dhingra-Kumar N, Kieny MP, Sheikh A (2017) Medication Without Harm: WHO's Third Global Patient Safety Challenge. The Lancet 389: 1680-1681.

36. G Jeetu, T Girish (2010) Prescription Drug Labeling Medication Errors: A Big Deal for Pharmacists. Journal of Young Pharmacists 2: 107-111.

37. Fialová D, Onder G (2009) Medication Errors in Elderly People: Contributing Factors and Future Perspectives. British Journal of Clinical Pharmacology 67: 641-645.

38. U.S. Food and Drug Administration Mixing Medications and Dietary Supplements Can Endanger Your Health.

39. Pierce CE, De Vries ST, Bodin-Parssinen S, Härmark L, Tregunno P, et al. (2019) Recommendations on the Use of Mobile Applications for the Collection and Communication of Pharmaceutical Product Safety Information: Lessons from IMI WEB-RADR. Drug Saf 42: 477-489.

40.(2018) European Medicines Agency Mobile Scanning and Other Technologies in the Labelling and Package Leaflet of Centrally Authorised Medicinal Products.

41. Mira JJ, Guilabert M, Carrillo I, Fernández C, Vicente MA, et al. (2015) Use of QR and EAN-13 Codes by Older Patients Taking Multiple Medications for a Safer Use of Medication. International Journal of Medical Informatics 84: 406-412.

42. Svensk J, McIntyre SE (2021) Using QR Code Technology to Reduce SelfAdministered Medication Errors. Journal of Pharmacy Practice 34: 587 591.

43. Batson S, Herranz A, Rohrbach N, Canobbio M, Mitchell SA, et al. (2021) Automation of In-Hospital Pharmacy Dispensing: A Systematic Review. Eur J Hosp Pharm 28: 58-64.

44. Hänninen K, Ahtiainen HK, Suvikas-Peltonen EM, Tötterman AM (2021) Automated Unit Dose Dispensing Systems Producing Individually Packaged and Labelled Drugs for Inpatients: A Systematic Review. Eur J Hosp Pharm.

45. Manias E, Kusljic S, Wu A (2020) Interventions to Reduce Medication Errors in Adult Medical and Surgical Settings: A Systematic Review. Therapeutic Advances in Drug Safety 11: 204209862096830.

46. Sabaté E (2003) Adherence to Long-Term Therapies. Evidence for Action.

47. MacCallum CA, Russo EB (2018) Practical Considerations in Medical Cannabis Administration and Dosing. European Journal of Internal Medicine 49: 12-19. 
48. Öblom H, Cornett C, Bøtker J, Frokjaer S, Hansen H, et al. (2020) DataEnriched Edible Pharmaceuticals (DEEP) of Medical Cannabis by Inkjet Printing. International Journal of Pharmaceutics 589: 119866.

49. Chao M, Öblom H, Cornett C, Bøtker J, Rantanen J, et al. (2021) DataEnriched Edible Pharmaceuticals (DEEP) with Bespoke Design, Dose and Drug Release. Pharmaceutics 13: 1866.

50. Tseng MH, Wu HC (2014) A Cloud Medication Safety Support System Using QR Code and Web Services for Elderly Outpatients. THC 22: 99113.

51. Nor RM, Mohamadali NA, Azmi, Marzuki A, Nor LM, et al. (2016) ScanMed: A Mobile Medicine Adherence Application with Intake Validation Using QR Code. In Proceedings of the 2016 6 $^{\text {th }}$ International Conference on Information and Communication Technology for The Muslim World (ICT4M), IEEE: Jakarta, Indonesia, pp. 112-117.

52. Sarzynski E, Decker B, Thul A, Weismantel D, Melaragni R, et al. (2017) Beta Testing a Novel Smartphone Application to Improve Medication Adherence. Telemedicine and e-Health 23: 339-348.

53. Capranzano P, Francaviglia B, Sardone A, Agnello F, Valenti N, et al. (2021) Suitability for Elderly with Heart Disease of a QR Code-Based Feedback of Drug Intake: Overcoming Limitations of Current Medication Adherence Telemonitoring Systems. International Journal of Cardiology 327: 209-216.

ISSN: 2574-1241

DOI: 10.26717/BJSTR.2022.41.006630

Achim Schneeberger. Biomed J Sci \& Tech Res

(C) (P) This work is licensed under Creative

Submission Link: https://biomedres.us/submit-manuscript.php
54. Rathbone AL, Prescott J (2017) The Use of Mobile Apps and SMS Messaging as Physical and Mental Health Interventions: Systematic Review. J Med Internet Res 19: e295.

55. Howells R (2019) Counterfeit Drugs: A Bitter Pill To Swallow. Forbes.

56. (2017) Center for Disease Control and Prevention Counterfeit Medicines. Travelers' Health.

57. (2014) World Customs Organization United Nations Launches Global Campaign Targeting the Criminal Counterfeit Trade.

58. Preis M, Breitkreutz J, Sandler N (2015) Perspective: Concepts of Printing Technologies for Oral Film Formulations. International Journal of Pharmaceutics 494: 578-584.

59. Fei J, Liu R (2016) Drug-Laden 3D Biodegradable Label Using QR Code for Anti-Counterfeiting of Drugs. Materials Science and Engineering: C 63: 657-662.

60. You M, Lin M, Wang S, Wang X, Zhang G, et al. (2016) Three-Dimensional Quick Response Code Based on Inkjet Printing of Upconversion Fluorescent Nanoparticles for Drug Anti-Counterfeiting. Nanoscale 8: 10096-10104.

61. Zhang H, Hua D, Huang C, Samal SK, Xiong R, et al. (2020) Materials and Technologies to Combat Counterfeiting of Pharmaceuticals: Current and Future Problem Tackling. Adv Mater 32: 1905486.

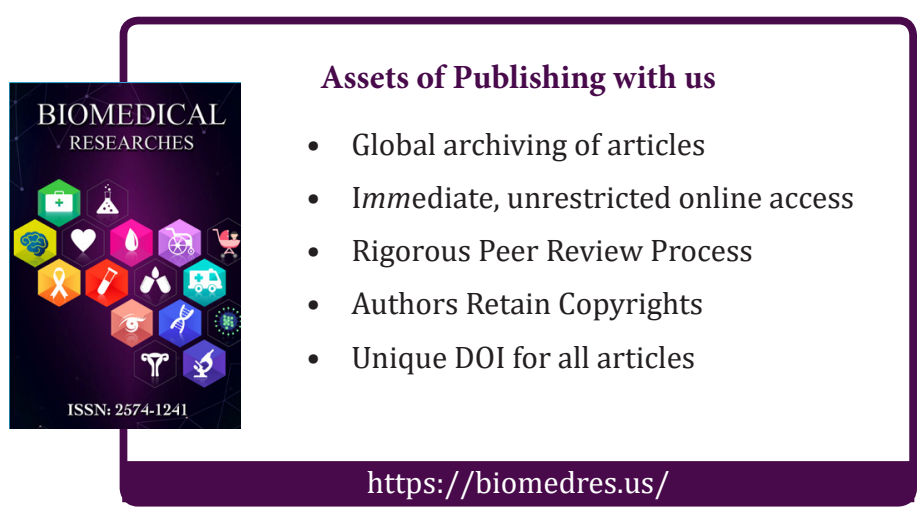

